TRANSACTIONS OF THE

AMERICAN MATHEMATICAL SOCIETY

Volume 350, Number 10, October 1998, Pages 4023-4040

S $0002-9947(98) 02076-5$

\title{
INFINITE TYPE HOMEOMORPHISMS OF THE CIRCLE AND CONVERGENCE OF FOURIER SERIES
}

\author{
ANTÔNIO ZUMPANO
}

\begin{abstract}
We consider the problem of convergence of Fourier series when we make a change of variable. Under a certain reasonable hypothesis, we give a necessary and sufficient condition for a homeomorphism of the circle to transform absolutely convergent Fourier series into uniformly convergent Fourier series.
\end{abstract}

\section{INTRODUCTION}

Let $A(T)$ be the space of all continuous functions of the circle $T$ with absolutely convergent Fourier series, and let $U(T)$ be the space of all continuous functions on the circle $T$ that have uniformly convergent Fourier series. If $\varphi$ is a homeomorphism of the circle $T$, we say that $\varphi$ transports $A(T)$ to $U(T)$ if $f \circ \varphi \in U(T)$ for all $f \in A(T)$.

A great deal of attention has been given to the following question: which homeomorphisms of the circle $T$ transport $A(T)$ to $U(T)$ ? We say that a homeomorphism $\varphi$ of the circle is of finite type if there is an integer $v$ with $v \geq 3$ such that: $\varphi$ is of class $C^{v}$ and $\left|\varphi^{\prime \prime}(t)\right|+\cdots+\left|\varphi^{(v)}(t)\right| \neq 0$ for all $t \in \mathbb{R}$. In 1974, R. Kaufman showed that if $\varphi$ is of finite type, then it transports $A(T)$ to $U(T)$. If $\varphi$ is an analytic homeromorphism of the circle, it is easy to see that either it is of finite type or $\varphi^{\prime \prime}(t)=0$ for all $t$. In the first case it transports $A(T)$ to $U(T)$ by the result of Kaufman; in the second case $\varphi$ transports $A(T)$ to $A(T)$, see [1]. But, not every $C^{\infty}$ homeomorphism of the circle transports $A(T)$ to $U(T)$, see [1] for the counterexample. We will be interested, therefore, in the case when $\varphi$ is of class $C^{\infty}$, but it may have a flat point, i.e. a point $t \in \mathbb{R}$ such that $\varphi^{(k)}(t)=0$ for all $k=2,3, \ldots$. (note that a $C^{\infty}$ homeomorphism with no flat point is of finite type). In a certain class of homeomorphisms of the circle, we will give a necessary and sufficient condition to transport $A(T)$ to $U(T)$.

\section{Statement of the Results}

The theorem of R. Kaufman that was mentioned above states that a homeomorphism $\varphi$ of the circle transports $A(T)$ to $U(T)$ if it is of finite type. The proof of this fact can be found in [1] and it is based on Lemma 2 below, but we suggest an alternative proof based on a result due to Stein and Wainger. See [2]. We state that result here as a lemma:

Received by the editors November 6, 1994 and, in revised form, October 23, 1996.

1991 Mathematics Subject Classification. Primary 42A20; Secondary 26A45.

Key words and phrases. Oscillatory integral, Fourier analysis, Fourier series, homeomorphism of the circle. 
Lemma 1 (Stein-Wainger). Let $p(t)$ be a real polynomial of degree $d$. Then,

$$
\left|\int_{-r}^{r} e^{i p(t)} \frac{1}{t} d t\right| \leq 6\left(2^{d+1}\right)-2 d-10 \text { for all } r>0 .
$$

They proved that lemma in a more general form in 1965 and the proof was published five years later [2].

The second lemma we state below was proved by R. Kaufman in 1974, see [1].

Lemma 2 (R. Kaufman). Let $f$ be a function of class $C^{k}$ on the interval $[-r, r]$ with $k \geq 2$. Suppose $1 \leq\left|f^{(k)}(t)\right| \leq b$ for all $t \in[-r, r]$. Then,

$$
\left|\int_{-r}^{r} e^{i f(t)} \frac{1}{t} d t\right| \leq C(k, b)
$$

where $C(k, b)$ is a constant that depends only on $k$ and $b$.

The fact is that Lemma 2 can be proved from Lemma 1 in a quite simple way. The proof of the second lemma given in [1] does not use Lemma 1 at all. Also, it is not difficult to see that Lemma 1 follows from Lemma 2 if we consider $d \geq 2$. So, they are indeed equivalent results.

The primary tool in dealing with oscillatory integrals as those in the lemmas is the Van der Corput lemma, see [3].

Our purpose is to deal with homeomorphisms of the circle not necessarily of finite type. To be precise, let $\varphi$ be a homeomorphism of a circle of class $C^{v}$, with $v \geq 3$, such that

$$
\left|\varphi^{\prime \prime}(t)\right|+\left|\varphi^{\prime \prime \prime}(t)\right|+\cdots+\left|\varphi^{(v)}(t)\right| \neq 0 \text { for all } t \neq 0, t \in[-\pi, \pi] .
$$

Suppose that there is a neighbourhood of zero, say $(-r, r)$ with $r<\pi$, such that:

$$
\begin{gathered}
\varphi \text { is an odd function on }(-r, r) . \\
\varphi^{\prime}(0)=0 \text { and } \varphi^{\prime \prime}(t)>0 \text { for all } t \in(0, r] .
\end{gathered}
$$

Also, assume that there is a constant $\theta$, with $0<\theta<1$ so that

$$
\begin{array}{r}
\varphi^{\prime}((1-\theta) a+\theta b) \leq \frac{1}{2}\left[\varphi^{\prime}(a)+\varphi^{\prime}(b)\right] \\
\text { for all } a, b \in[0, r) \text { with } a \leq b .
\end{array}
$$

We describe (4) by saying that $\varphi^{\prime}$ has uniform bounded doubling time. In particular $\varphi^{\prime}$ has what is called bounded doubling time, for if we put $a=0$ above, we have that $\varphi^{\prime}(\theta t) \leq \frac{1}{2} \varphi^{\prime}(t)$ for all $t \in[0, r)$. Also, condition (4) with $\theta=\frac{1}{2}$ means that $\varphi^{\prime}$ is a convex function on [0,r); but it does not imply convexity of $\varphi^{\prime}$ (see [5] for an example of a homeomorphism $\varphi$ of the circle that satisfies all the four conditions and $\varphi^{\prime}$ is not convex in any interval of the form $\left.(0, r), r>0\right)$.

We shall prove the following result:

Theorem 1. Let $\varphi$ be a homeomorphism of the circle of class $C^{v}, v \geq 3$. Suppose that $\varphi$ satisfies (1), (2), (3) and (4). Then, $\varphi$ transports $A(T)$ to $U(T)$ if and only if there are constants $\zeta, M$ and $\lambda$ with $0<\zeta<1, M>0$ and $\lambda>1$ such that

$$
\frac{\varphi^{\prime}(b)-\varphi^{\prime}((1-\zeta) a+\zeta b)}{\varphi^{\prime}((1-\zeta) a+\zeta b)-\varphi^{\prime}(a)} \leq M
$$

whenever $\varphi^{\prime}((1-\zeta) a+\zeta b) \leq \lambda \varphi^{\prime}(a)$, for all $0<a<b<r$. 
An easy way to verify condition (5) is that: suppose $\varphi^{\prime}(0)=0$ and $\varphi^{\prime \prime}(t)>0$ for all $t \in(0, r)$; then all three conditions below imply condition (5). Moreover, they are related in the following way: $(i) \Rightarrow($ ii $) \Longleftrightarrow$ (iii), where

(i) $\log \circ \varphi^{\prime}(t)$ is a concave function on $(0, r)$.

(ii) There is $\zeta$, with $0<\zeta<1$, such that for all $a, b \in(0, r)$ with $a \leq b$,

$$
\varphi^{\prime}((1-\zeta) a+\zeta b) \geq \sqrt{\varphi^{\prime}(a)} \sqrt{\varphi^{\prime}(b)} .
$$

(iii) There are $\zeta$ and $\sigma$ with $0<\zeta<1,0<\sigma<1$ such that for all $a, b \in(0, r)$ with $a \leq b$ we have

$$
\varphi^{\prime}((1-\zeta) a+\zeta b) \geq\left[\varphi^{\prime}(a)\right]^{1-\sigma}\left[\varphi^{\prime}(b)\right]^{\sigma} .
$$

To see that condition (iii) implies (5) we use the mean value theorem to get

$$
\begin{gathered}
\frac{\varphi^{\prime}(b)-\varphi^{\prime}((1-\zeta) a+\zeta b)}{\varphi^{\prime}((1-\zeta) a+\zeta b)-\varphi^{\prime}(a)} \\
\leq \frac{\varphi^{\prime}((1-\zeta) a+\zeta b)\left[\varphi^{\prime}((1-\zeta) a+\zeta b)^{\frac{1-\sigma}{\sigma}}-\varphi^{\prime}(a)^{\frac{1-\sigma}{\sigma}}\right]}{\varphi^{\prime}(a)^{\frac{1-\sigma}{\sigma}}\left[\varphi^{\prime}((1-\zeta) a+\zeta b)-\varphi^{\prime}(a)\right]} \\
\leq \frac{1-\sigma}{\sigma}\left[\frac{\varphi^{\prime}((1-\zeta) a+\zeta b)}{\varphi^{\prime}(a)}\right]^{\frac{1-\sigma}{\sigma}} \quad \text { for } \quad \sigma<\frac{1}{2} .
\end{gathered}
$$

Also, assuming (iii), we can prove by induction that

$$
\varphi^{\prime}\left((1-\zeta)^{k} a+\left[1-(1-\zeta)^{k}\right] b\right) \geq\left[\varphi^{\prime}(a)\right]^{(1-\sigma)^{k}}\left[\varphi^{\prime}(b)\right]^{1-(1-\sigma)^{k}} \text { for all } k \in \mathbb{N} .
$$

So, (iii) implies (ii). Condition (ii) does not imply (i), see [5].

Using the theorem we can see that homeomorphisms of the circle as $\varphi^{\prime}(t)=e^{-1 / t}$ or $\varphi^{\prime}(t)=e^{-1 / t^{2}}$ for $t \in(0,1 / 4)$, transport $A(T)$ to $U(T)$.

In [5] there is an example of a $C^{\infty}$ homeomorphism $\varphi$ of the circle that satisfies conditions (1), (2), (3) and (4) but does not satisfy condition (5).

\section{Proof of the theorem}

The space $A(T)$ is a Banach space with the norm

$$
\|f\|_{A(T)}=\sum_{n \in \mathbb{Z}}\left|\hat{f}_{n}\right|, \text { where } \hat{f}_{n}=\frac{1}{2 \pi} \int_{-\pi}^{\pi} f(t) e^{-i n t} d t, n \in \mathbb{Z},
$$

are the Fourier coefficients of $f$. Also, $U(T)$ is a Banach space with the norm

$$
\|f\|_{U(T)}=\sup \left\{\left|S_{m}(f, x)\right| ; x \in[-\pi, \pi], m=0,1,2, \ldots .\right\},
$$

where $S_{m}(f, x)=\sum_{-m}^{m} \hat{f}_{n} e^{i n x}$ are the partial sums of the Fourier series of $f$.

A homeomorphism of the circle $\varphi$ of class $C^{k}, k \geq 1$, transports $A(T)$ to $U(T)$ if and only if there is a constant $C$, that does not depend on $m, n$ or $x$, such that

$$
\begin{aligned}
\left|\int_{-\pi}^{\pi} e^{i n \varphi(t+x)} D_{m}(t) d t\right| & \leq C \\
\text { for all } m & =0,1,2, \ldots, n \in \mathbb{Z} \text { and } x \in[-\pi, \pi],
\end{aligned}
$$


where $D_{m}(t)$ is the Dirichlet Kernel, i.e.

$$
D_{m}(t)=\frac{\sin \left(m+\frac{1}{2}\right) t}{\sin \left(\frac{t}{2}\right)}=\frac{2 \sin m t}{t}+\mathcal{O}(1)
$$

on any compact subset of $(-2 \pi, 2 \pi)$.

Let $(-\tau, \tau)$ be a small neighbourhood of zero and suppose that $\tau \leq|x| \leq \pi$. By (1), there is $\delta>0$ such that: if $\tau \leq|x| \leq \pi$, then, for some $k$, depending on $x$, with $2 \leq k \leq v$ we have $\left|\varphi^{(k)}(t+x)\right| \geq \delta$ for all $t$ with $|t| \leq \delta$. Therefore, as in reference [1], the integral in (6) is bounded for $\tau \leq|x| \leq \pi$. So, $\varphi$ transports $A(T)$ to $U(T)$ if and only if

$$
\begin{aligned}
& \left|\int_{-\pi}^{\pi} e^{i n \varphi(t)} \frac{\sin m(t-x)}{t-x} d t\right| \leq C \\
& \quad \text { for all } n \in \mathbb{Z}, m=0,1,2, \ldots \text { and } x \in(-\tau, \tau),
\end{aligned}
$$

where $\tau$ is any positive number less than or equal to $\frac{\pi}{2}$ and $C$ is a constant that does not depend on $n, m$ and $x$. ( The number $\tau$ will be chosen conveniently and it will depend on the constant $\theta$.) Let $\tau$ be a number such that $\tau<\frac{r}{2}<\frac{\pi}{2}$. So,

$$
\left|\int_{-\pi}^{\pi} e^{i n \varphi(t)} \frac{\sin m(t-x)}{t-x} d t\right| \leq\left|\int_{-r}^{r} e^{i n \varphi(t)} \frac{\sin m(t-x)}{t-x} d t\right|+\frac{4 \pi}{r}
$$

for all $n \in \mathbb{Z}, m=0,1,2, \ldots$ and for all $x \in(-\tau, \tau)$.

Also, since $\varphi$ is odd on $(-r, r)$, then, if we prove that the integral

$$
\int_{0}^{r} e^{i n \varphi(t)} \frac{\sin m(t+x)}{t+x} d t
$$

is bounded for all $x \in[0, \tau), n>0$ and $m>0$, we conclude that (7) holds if and only if the integral

$$
\int_{0}^{r} e^{i n \varphi(t)} \frac{\sin m(t-x)}{t-x} d t
$$

is bounded for all $x \in(0, \tau), n>0$ and $m>0$.

The boundedness of the integral (8) was proved by Nagel, Vance, Wainger and Weinberg in the second paragraph of [4]. The proof does not use the full force of condition (4); it is based on the bounded doubling time only.

The main part of the theorem is the question of boundedness of the integral (9) for $x \in(0, \tau)$ and $n>0, m>0$.

To get boundedness of the integral (9) we have to deal with two major problems: the first one is that the derivative of $m t-n \varphi(t)$ may vanish somewhere between zero and $r$; and in that case we do not have enough oscillation around the vanishing point. The second one is that we have a bad singularity at the point $x$, and around that point, oscillation will not be enough to bound the integral. But, condition (5) will tell us whether there is cancellation around the point $x$ or not. So, to estimate the integral (9) we deal simultaneously with oscillation and cancellation.

Put $f(t)=m t-n \varphi(t)$ and suppose that $x \leq \frac{1}{m}$. Assume first that $f^{\prime}(r)=$ $m-n \varphi^{\prime}(r) \geq 0$. Then, we have two possibilities: the first one is $\theta r \leq \frac{2}{m}$. If so, (9) is bounded by $\frac{2}{\theta}$. The second one is $\theta r>\frac{2}{m}$, and in that case we divide the integral 
in three parts: over $\left[0, \frac{2}{m}\right]$ it is bounded by 2 , over $[\theta r, r]$ by $\frac{2}{\theta}$ and over $\left[\frac{2}{m}, \theta r\right]$ we have

$$
\begin{gathered}
\quad\left|\int_{2 / m}^{\theta r} e^{i n \varphi(t)} \frac{\sin m(t-x)}{t-x} d t\right| \\
\leq\left|\int_{2 / m}^{\theta r} e^{i(m t+n \varphi(t))} \frac{1}{t-x} d t\right|+\left|\int_{2 / m}^{\theta r} e^{i f(t)} \frac{1}{t-x} d t\right| \\
\leq \frac{4}{m\left(\frac{2}{m}-x\right)}+\frac{4}{\left[m-n \varphi^{\prime}(\theta r)\right]\left(\frac{2}{m}-x\right)} \leq 4+\frac{4 m}{\left[m-n \frac{1}{2} \varphi^{\prime}(r)\right]} \leq 12 .
\end{gathered}
$$

We used the Van der Corput lemma, the bounded doubling time, the monotonicity of $\varphi^{\prime}$ and the assumption $m-n \varphi^{\prime}(r) \geq 0$ to obtain the above estimates. We have not used the full force of condition (4). Now, let's assume that $f^{\prime}(r)=m-n \varphi^{\prime}(r)<0$. Then, there is a number $\xi$ with $0<\xi<r$ such that $f^{\prime}(\xi)=0$. This means that $\varphi^{\prime}(\xi)=\frac{m}{n}$. As before, we have two possibilites: $\theta \xi \leq \frac{2}{m}$ or $\frac{2}{m}<\theta \xi$. If $\theta \xi \leq \frac{2}{m}$, then $\xi<\frac{1}{\theta} \xi \leq \frac{1}{\theta^{2}} \frac{2}{m}$. We can assume that $\frac{1}{\theta^{2}} \frac{2}{m}<r$, because otherwise, (9) is bounded by $m r \leq \frac{2}{\theta^{2}}$. So, assuming $\frac{2}{\theta^{2} m}<r$ we divide the integral in two parts: over $\left[0, \frac{2}{\theta^{2} m}\right]$ it is bounded by $\frac{2}{\theta^{2}}$, and over $\left[\frac{2}{\theta^{2} m}, r\right]$ we have

$$
\begin{gathered}
\left|\int_{2 / \theta^{2} m}^{r} e^{i n \varphi(t)} \frac{\sin m(t-x)}{t-x} d t\right| \\
\leq\left|\int_{2 / \theta^{2} m}^{r} e^{i(m t+n \varphi(t))} \frac{1}{t-x} d t\right|+\left|\int_{2 / \theta^{2} m}^{r} e^{i f(t)} \frac{1}{t-x} d t\right| \\
\leq \frac{4}{m\left(\frac{2}{\theta^{2} m}-x\right)}+\frac{4}{\left[n \varphi^{\prime}\left(\frac{2}{\theta^{2} m}\right)-m\right]\left(\frac{2}{\theta^{2} m}-x\right)} \\
\leq 4+\frac{4 m}{\left[n \varphi^{\prime}\left(\frac{\xi}{\theta}\right)-m\right]} \leq 4+\frac{4 m}{2 n \varphi^{\prime}(\xi)-m}=8 .
\end{gathered}
$$

If $\frac{2}{m}<\theta \xi$, then we do the following: the integral is bounded by 2 over the interval $\left[0, \frac{2}{m}\right]$ and

$$
\begin{aligned}
& \left|\int_{2 / m}^{\theta \xi} e^{i n \varphi(t)} \frac{\sin m(t-x)}{t-x} d t\right| \leq 4+\left|\int_{2 / m}^{\theta \xi} e^{i f(t)} \frac{1}{t-x} d t\right| \\
& \leq 4+\frac{4}{\left[m-n \varphi^{\prime}(\theta \xi)\right]\left(\frac{2}{m}-x\right)} \leq 4+\frac{4 m}{\left[m-\frac{1}{2} n \varphi^{\prime}(\xi)\right]}=12 .
\end{aligned}
$$

Now, if $\frac{\xi}{\theta} \geq r$, then

$$
\left|\int_{\theta \xi}^{r} e^{i n \varphi(t)} \frac{\sin m(t-x)}{t-x} d t\right| \leq \frac{r}{\theta \xi-x} \leq \frac{\frac{\xi}{\theta}}{\frac{1}{2} \theta \xi}=\frac{2}{\theta^{2}}
$$

So, we may assume $\frac{\xi}{\theta}<r$. In that case we have

$$
\left|\int_{\theta \xi}^{\xi / \theta} e^{i n \varphi(t)} \frac{\sin m(t-x)}{t-x} d t\right| \leq \frac{\frac{\xi}{\theta}}{\theta \xi-x} \leq \frac{2}{\theta^{2}}
$$


and

$$
\begin{gathered}
\left|\int_{\xi / \theta}^{r} e^{i n \varphi(t)} \frac{\sin m(t-x)}{t-x} d t\right| \leq 4+\frac{4}{\left[n \varphi^{\prime}\left(\frac{\xi}{\theta}\right)-m\right]\left(\frac{\xi}{\theta}-x\right)} \\
\leq 4+\frac{4 m}{2 n \varphi^{\prime}(\xi)-m}=8 .
\end{gathered}
$$

Again we used the Van der Corput lemma and the bounded doubling time to obtain the above estimates. Hence, the integral (9) is bounded if $x \leq \frac{1}{m}$.

Let's consider now the case when $x>\frac{1}{m}$. We have that (7) holds if and only if

$$
\left|\int_{0}^{x-\frac{1}{m}} e^{i f(t)} \frac{1}{t-x} d t+\int_{x+\frac{1}{m}}^{r} e^{i f(t)} \frac{1}{t-x} d t\right| \leq C
$$

for all $x \in(0, \tau), m, n \in \mathbb{N}$ with $\frac{1}{m}<x$. (C is of course a constant that does not depend on $x, m$ and $n$.) So, we will prove that (10) holds if and only if the homeomorphism $\varphi$ satisfies condition (5).

Let's prove first that condition (5) is necessary. For this, let $\left(\zeta_{k}\right)_{k \in \mathbb{N}}$ and $\left(\lambda_{k}\right)_{k \in \mathbb{N}}$ be sequences such that,

$$
0<\zeta_{k}<1, \lambda_{k}>1 \text { for all } k \in \mathbb{N} \text { and } \zeta_{k} \rightarrow 1, \lambda_{k} \rightarrow 1 \text {. }
$$

Suppose that $\varphi$ does not satisfy condition (5). Then, for each $k \in \mathbb{N}$ there exist numbers $a_{k}$ and $b_{k}$, with $0<a_{k}<b_{k}<\frac{1}{k} r$, so that

$$
\frac{\varphi^{\prime}\left(b_{k}\right)-\varphi^{\prime}\left(x_{k}\right)}{\varphi^{\prime}\left(x_{k}\right)-\varphi^{\prime}\left(a_{k}\right)}>\frac{\zeta_{k}}{1-\zeta_{k}}
$$

and

$$
\varphi^{\prime}\left(x_{k}\right) \leq \lambda_{k} \varphi^{\prime}\left(a_{k}\right)
$$

where $x_{k}=\left(1-\zeta_{k}\right) a_{k}+\zeta_{k} b_{k}$. Put

$$
\begin{aligned}
\epsilon_{k} & =\zeta_{k}\left(b_{k}-a_{k}\right), \\
\epsilon_{k}^{\prime} & =\left(1-\zeta_{k}\right)\left(b_{k}-a_{k}\right), \\
\nu_{k} & =\frac{1}{\epsilon_{k}\left[\varphi^{\prime}\left(x_{k}\right)-\varphi^{\prime}\left(a_{k}\right)\right]}, \\
\mu_{k} & =\varphi^{\prime}\left(x_{k}\right) \nu_{k} .
\end{aligned}
$$

Let $m_{k}$ be the smallest integer greater than $\mu_{k}$ and $n_{k}$ be the greatest integer less than $\nu_{k}$. So, by (11), for $k$ sufficiently large we have

$$
\frac{1}{2} \leq \epsilon_{k}\left[m_{k}-n_{k} \varphi^{\prime}\left(x_{k}-\epsilon_{k}\right)\right] \leq \frac{3}{2}
$$

and

$$
\epsilon_{k}^{\prime}\left[n_{k} \varphi^{\prime}\left(x_{k}+\epsilon_{k}^{\prime}\right)-m_{k}\right]>\frac{1}{2} .
$$

Inequality (14) implies that there is $\overline{\epsilon_{k}}$ with $0<\overline{\epsilon_{k}}<\epsilon_{k}^{\prime}$ such that

$$
\overline{\epsilon_{k}}\left[n_{k} \varphi^{\prime}\left(x_{k}+\overline{\epsilon_{k}}\right)-m_{k}\right]=\frac{1}{2} .
$$

Also, by (12), $\epsilon_{k} m_{k} \rightarrow+\infty$ and since $x_{k}>\epsilon_{k}$, then $x_{k}>\frac{1}{m_{k}}$. Since $x_{k} \rightarrow 0$, then $0<x_{k}<\tau$ for $k$ large enough.

Suppose that $\overline{\epsilon_{k}} \leq \frac{1}{m_{k}}$ for an infinite number of indices $k$. 
Let $f_{k}(t)=m_{k} t-n_{k} \varphi(t)$. Then, by (15) and the Van der Corput lemma we have that

$$
\left|\int_{x_{k}+\frac{1}{m_{k}}}^{r} e^{i f_{k}(t)} \frac{1}{t-x_{k}} d t\right| \leq \frac{4 m_{k}}{\left|f_{k}^{\prime}\left(x_{k}+\bar{\varepsilon}_{k}\right)\right|} \leq \frac{4}{\bar{\varepsilon}_{k}\left|f_{k}^{\prime}\left(x_{k}+\bar{\varepsilon}_{k}\right)\right|}=8
$$

for an infinite number of indices $k$. By (13) and the Van der Corput lemma,

$$
\left|\int_{0}^{x_{k}-\varepsilon_{k}} e^{i f_{k}(t)} \frac{1}{t-x_{k}} d t\right| \leq \frac{4}{\varepsilon_{k} f_{k}^{\prime}\left(x_{k}-\varepsilon_{k}\right)} \leq 8 .
$$

Now, using (13),

$$
\begin{aligned}
\left|\int_{x_{k}-\varepsilon_{k}}^{x_{k}-\frac{1}{m_{k}}}\left[e^{i f_{k}(t)}-e^{i f_{k}\left(x_{k}\right)}\right] \frac{1}{t-x_{k}} d t\right| & \leq \quad \varepsilon_{k} f_{k}^{\prime}\left(x_{k}-\varepsilon_{k}\right) \leq \frac{3}{2} \\
\text { and } & \\
\left|\int_{x_{k}-\varepsilon_{k}}^{x_{k}-\frac{1}{m_{k}}} e^{i f_{k}\left(x_{k}\right)} \frac{1}{t-x_{k}} d t\right| & =\int_{1 / m_{k}}^{\varepsilon_{k}} \frac{1}{t} d t=\log \left(\varepsilon_{k} m_{k}\right) .
\end{aligned}
$$

Hence, we conclude that (10) does not hold. Suppose now that $\bar{\varepsilon}_{k}>\frac{1}{m_{k}}$ for all $k$ large enough. Then, by (13) and (15),

$$
\begin{aligned}
\left|\int_{x_{k}+\bar{\varepsilon}_{k}}^{r} e^{i f_{k}(t)} \frac{1}{t-x_{k}} d t\right| & \leq \frac{4}{\bar{\varepsilon}_{k}\left|f_{k}^{\prime}\left(x_{k}+\bar{\varepsilon}_{k}\right)\right|}=8 \\
& \text { and } \\
\left|\int_{0}^{x_{k}-\varepsilon_{k}} e^{i f_{k}(t)} \frac{1}{t-x_{k}} d t\right| & \leq \frac{4}{\varepsilon_{k} f_{k}^{\prime}\left(x_{k}-\varepsilon_{k}\right)} \leq 8,
\end{aligned}
$$

for all $k$ large enough. Finally, by (13) and (15),

$$
\left|\int_{x_{k}-\varepsilon_{k}}^{x_{k}-\frac{1}{m_{k}}}\left(e^{i f_{k}(t)}-e^{i f_{k}\left(x_{k}\right)}\right) \frac{1}{t-x_{k}} d t\right| \leq \varepsilon_{k} f_{k}^{\prime}\left(x_{k}-\varepsilon_{k}\right) \leq \frac{3}{2}
$$

and

$$
\left|\int_{x_{k}+\frac{1}{m_{k}}}^{x_{k}+\bar{\varepsilon}_{k}}\left(e^{i f_{k}(t)}-e^{i f_{k}\left(x_{k}\right)}\right) \frac{1}{t-x_{k}} d t\right| \leq \varepsilon_{k} f_{k}^{\prime}\left(x_{k}-\varepsilon_{k}\right) \leq \frac{3}{2} .
$$

Hence, since

$$
\begin{gathered}
\left|\int_{x_{k}-\varepsilon_{k}}^{x_{k}-\frac{1}{m_{k}}} e^{i f_{k}\left(x_{k}\right)} \frac{1}{t-x_{k}} d t+\int_{x_{k}+\frac{1}{m_{k}}}^{x_{k}+\bar{\varepsilon}_{k}} e^{i f_{k}\left(x_{k}\right)} \frac{1}{t-x_{k}}\right| \\
=\left|\int_{-\varepsilon_{k}}^{-\frac{1}{m_{k}}} \frac{1}{t} d t+\int_{1 / m_{k}}^{\bar{\varepsilon}_{k}} \frac{1}{t} d t\right|=\log \left(\frac{\varepsilon_{k}}{\bar{\varepsilon}_{k}}\right) \geq \log \left(\frac{\varepsilon_{k}}{\varepsilon_{k}^{\prime}}\right)=\log \left(\frac{\zeta_{k}}{1-\zeta_{k}}\right),
\end{gathered}
$$

we conclude that (10) does not hold. Therefore, condition (5) is necessary. 
Now, let's prove sufficiency. So, assuming that $\varphi$ satisfies condition (5), we have to show that (10) is true. For this, let $x \in(0, \tau), n, m \in \mathbb{N}$ with $x>\frac{1}{m}, m>0, n>0$. We said before that the number $\tau$ would be chosen conveniently. So, let $\tau<\frac{1}{2} \theta r$. (The reason for that choice will be clear in the proof that follows. It is not quite necessary, but it simplifies the proof a lot.) Put $f(t)=m t-n \varphi(t)$ as before and suppose that $f^{\prime}(r)=m-n \varphi^{\prime}(r) \geq 0$. Since $x+\frac{1}{m}<2 x<2 \tau<\theta r$, then using the bounded doubling time, the monotonicity of $\varphi^{\prime}$, and the Van der Corput lemma we have that

$$
\begin{aligned}
\left|\int_{0}^{x-\frac{1}{m}} e^{i f(t)} \frac{1}{t-x} d t\right| & \leq \frac{4 m}{f^{\prime}\left(x-\frac{1}{m}\right)} \leq \frac{4 m}{f^{\prime}(\theta r)} \leq \frac{4 m}{m-n \frac{1}{2} \varphi^{\prime}(r)} \leq 8, \\
\left|\int_{x+\frac{1}{m}}^{\theta r} e^{i f(t)} \frac{1}{t-x} d t\right| & \leq \frac{4 m}{f^{\prime}(\theta r)} \leq 8
\end{aligned}
$$

and

$$
\left|\int_{\theta r}^{r} e^{i f(t)} \frac{1}{t-x} d t\right| \leq \frac{r}{\theta r-x} \leq \frac{2}{\theta} .
$$

Hence, (10) holds if $f^{\prime}(r)=m-n \varphi^{\prime}(r) \geq 0$ and $\frac{1}{m}<x<\tau$.

Suppose now that $f^{\prime}(r)=m-n \varphi^{\prime}(r)<0$. So, there exists a number $\xi$ with $0<\xi<r$ such that $f^{\prime}(\xi)=m-n \varphi^{\prime}(\xi)=0$. Since $m x>1$, then there exists a number $\varepsilon$ with $0<\varepsilon<x$ such that $\varepsilon\left(m-n \varphi^{\prime}(x-\varepsilon)\right)=1-\frac{1}{\lambda}$. We have two cases to consider: one is when $x \leq \xi$ and the other is when $\xi<x$. We will treat each one of them separately. From now on we have to deal simultaneously with oscillation and cancellation. Roughly, the problem is that: when we approach near $x$, the singularity is so bad that even if there is oscillation at $x$, this is not enough to guarantee boundedness. So, we have to use the cancellation we have around $x$. In order to have an effective cancellation we need to use condition (5). On the other hand, when we approach $\xi$, we don't have oscillation since $f^{\prime}(\xi)=0$. So, to deal with the integral around $\xi$ we have to use condition (4). An important factor will be how far (with respect to $\varepsilon$ ) is $\xi$ from $x$.

Assume that $x \leq \xi$. Call $\alpha=\xi-x$. The arguments we will use depend on the relation between $\varepsilon, \alpha$ and $m$. Suppose we have the following situation: $0<\frac{1}{m}<$ $\varepsilon<\frac{1}{2} \theta \alpha$. Let's choose a number $A$ such that $\alpha+\varepsilon=\theta(A+\alpha+\varepsilon)$. We see that $A=\frac{1-\theta}{\theta}(\alpha+\varepsilon)$ is that number. We are going to divide the integral in several parts and prove boundedness of each one separately:

$$
\begin{gathered}
\left|\int_{0}^{x-\varepsilon} e^{i f(t)} \frac{1}{t-x} d t\right| \leq \frac{4}{\varepsilon f^{\prime}(x-\varepsilon)}=\frac{4 \lambda}{\lambda-1}, \\
\left|\int_{x-\varepsilon}^{x-\frac{1}{m}} e^{i f(t)} \frac{1}{t-x} d t+\int_{x+\frac{1}{m}}^{x+\varepsilon} e^{i f(t)} \frac{1}{t-x} d t\right| \\
=\mid \int_{x-\varepsilon}^{x-\frac{1}{m}}\left(e^{i f(t)}-e^{i f(x)} \frac{1}{t-x} d t+\int_{x+\frac{1}{m}}^{x+\varepsilon}\left(e^{i f(t)}-e^{i f(x)}\right) \frac{1}{t-x} d t \mid\right. \\
\leq 2 \varepsilon f^{\prime}(x-\varepsilon)=\frac{2(\lambda-1)}{\lambda} .
\end{gathered}
$$


To estimate the integral from $x+\varepsilon$ to $x+\frac{1}{2} \theta \alpha$ we use condition (4). This condition assures enough oscillation in that interval. We claim that

$$
m-n \varphi^{\prime}\left(x+\frac{1}{2} \theta \alpha\right) \geq \frac{1}{2}\left[m-n \varphi^{\prime}(x-\varepsilon)\right] .
$$

To prove (16) we use condition (4): since $\varepsilon<\frac{1}{2} \theta \alpha$, then

$$
x+\frac{1}{2} \theta \alpha \leq(1-\theta)(x-\varepsilon)+\theta \xi
$$

this implies that

$$
\varphi^{\prime}\left(x+\frac{1}{2} \theta \alpha\right) \leq \varphi^{\prime}((1-\theta)(x-\varepsilon)+\theta \xi) \leq \frac{1}{2}\left(\varphi^{\prime}(x-\varepsilon)+\varphi^{\prime}(\xi)\right)
$$

and (16) follows. Therefore, by (16)

$$
\left|\int_{x+\varepsilon}^{x+\frac{1}{2} \theta \alpha} e^{i f(t)} \frac{1}{t-x} d t\right| \leq \frac{4}{\varepsilon f^{\prime}\left(x+\frac{1}{2} \theta \alpha\right)} \leq \frac{8}{\varepsilon f^{\prime}(x-\varepsilon)}=\frac{8 \lambda}{\lambda-1} .
$$

Now, we have to pass through the point $\xi$ where we don't have oscillation at all. But since we are away from $x$, things become much simpler:

$$
\left|\int_{x+\frac{1}{2} \theta \alpha}^{\xi+A} e^{i f(t)} \frac{1}{t-x} d t\right| \leq \log \left(\frac{\xi+A-x}{\frac{1}{2} \theta \alpha}\right) \leq \log \left(\frac{2+\theta(1-\theta)}{\theta^{2}}\right) .
$$

Note that we can assume that $\xi+A<r$, because otherwise,

$$
\left|\int_{x+\frac{1}{2} \theta \alpha}^{r} e^{i f(t)} \frac{1}{t-x} d t\right| \leq \log \left(\frac{r-x}{\frac{1}{2} \theta \alpha}\right) \leq \log \left(\frac{\xi+A-x}{\frac{1}{2} \theta \alpha}\right)
$$

and we are done. Finally, to evaluate the integral from $\xi+A$ to $r$ we use again condition (4). This condition, as before, assures enough ocillation in that interval. We claim that

$$
n \varphi^{\prime}(\xi+A)-m \geq m-n \varphi^{\prime}(x-\varepsilon) .
$$

By the choice of $A$, we have that $\xi=(1-\theta)(x-\varepsilon)+\theta(\xi+A)$. So,

$$
\varphi^{\prime}(\xi) \leq \frac{1}{2}\left(\varphi^{\prime}(x-\varepsilon)+\varphi^{\prime}(\xi+A)\right)
$$

and (17) follows. Using (17) now we have

$$
\begin{aligned}
\left|\int_{\xi+A}^{r} e^{i f(t)} \frac{1}{t-x} d t\right| & \leq \frac{4}{(\xi+A-x)\left|f^{\prime}(\xi+A)\right|}=\frac{4}{(\alpha+A)\left|f^{\prime}(\xi+A)\right|} \\
& \leq \frac{4}{\varepsilon f^{\prime}(x-\varepsilon)}=\frac{4 \lambda}{\lambda-1}
\end{aligned}
$$

since $\alpha+A>\alpha>\varepsilon$.

Let's see now another possible situation: suppose that $0<\varepsilon \leq \frac{1}{m}<\frac{1}{2} \theta \alpha$. Then,

$$
\begin{aligned}
\left|\int_{0}^{x-\frac{1}{m}} e^{i f(t)} \frac{1}{t-x} d t\right| & \leq \frac{4 m}{f^{\prime}\left(x-\frac{1}{m}\right)} \leq \frac{4}{\varepsilon f^{\prime}(x-\varepsilon)}=\frac{4 \lambda}{\lambda-1} \\
\left|\int_{x+\frac{1}{m}}^{x+\frac{1}{2} \theta \alpha} e^{i f(t)} \frac{1}{t-x} d t\right| & \leq \frac{4 m}{f^{\prime}\left(x+\frac{1}{2} \theta \alpha\right)} \leq \frac{8}{\varepsilon f^{\prime}(x-\varepsilon)}=\frac{8 \lambda}{\lambda-1},
\end{aligned}
$$

by (16).

From $x+\frac{1}{2} \theta \alpha$ to $\xi+A$ and from $\xi+A$ to $r$ there is no change. 
If $0<\varepsilon<\frac{1}{2} \theta \alpha \leq \frac{1}{m}$, then

$$
\left|\int_{0}^{x-\frac{1}{m}} e^{i f(t)} \frac{1}{t-x} d t\right| \leq \frac{4 m}{f^{\prime}\left(x-\frac{1}{m}\right)} \leq \frac{4}{\varepsilon f^{\prime}(x-\varepsilon)}=\frac{4 \lambda}{\lambda-1} .
$$

If $x+\frac{1}{m}<\xi+A$, then

$$
\begin{aligned}
\left|\int_{x+\frac{1}{m}}^{\xi+A} e^{i f(t)} \frac{1}{t-x} d t\right| & \leq \log \left(\frac{\xi+A-x}{\frac{1}{m}}\right) \leq \log \left(\frac{\alpha+A}{\frac{1}{2} \theta \alpha}\right) \\
& \leq \log \left(\frac{2+\theta(1-\theta)}{\theta^{2}}\right) .
\end{aligned}
$$

Again we can assume $\xi+A<r$. Note that $x+\frac{1}{m}<r$ because $\frac{1}{m}<x<\tau<\frac{r}{2}$.

$$
\left|\int_{\xi+A}^{r} e^{i f(t)} \frac{1}{t-x} d t\right| \leq \frac{4 \lambda}{\lambda-1} \quad \text { as before. }
$$

If $\xi+A \leq x+\frac{1}{m}$, then

$$
\begin{aligned}
\left|\int_{x+\frac{1}{m}}^{r} e^{i f(t)} \frac{1}{t-x} d t\right| & \leq \frac{4 m}{\left|f^{\prime}\left(x+\frac{1}{m}\right)\right|} \leq \frac{4}{\varepsilon\left|f^{\prime}(\xi+A)\right|} \\
& \leq \frac{4}{\varepsilon f^{\prime}(x-\varepsilon)}=\frac{4 \lambda}{\lambda-1}
\end{aligned}
$$

by (17).

The next situation is $\frac{1}{2} \theta \alpha \leq \varepsilon$ and $\frac{1}{m} \geq \varepsilon$. Assuming this we have

$$
\left|\int_{0}^{x-\frac{1}{m}} e^{i f(t)} \frac{1}{t-x} d t\right| \leq \frac{4 m}{f^{\prime}\left(x-\frac{1}{m}\right)} \leq \frac{4}{\varepsilon f^{\prime}(x-\varepsilon)}=\frac{4 \lambda}{\lambda-1} .
$$

If $x+\frac{1}{m}<\xi+A$, then

$$
\left|\int_{x+\frac{1}{m}}^{\xi+A} e^{i f(t)} \frac{1}{t-x} d t\right| \leq \log \left(\frac{\alpha+A}{\frac{1}{m}}\right) \leq \log \left(\frac{2+\theta(1-\theta)}{\theta^{2}}\right) .
$$

As before, we can assume $\xi+A<r$, so

$$
\left|\int_{\xi+A}^{r} e^{i f(t)} \frac{1}{t-x} d t\right| \leq \frac{4}{(\alpha+A)\left|f^{\prime}(\xi+A)\right|} \leq \frac{4}{\varepsilon f^{\prime}(x-\varepsilon)}=\frac{4 \lambda}{\lambda-1},
$$

by (17).

If $\xi+A \leq x+\frac{1}{m}$, then, by (17), the integral from $x+\frac{1}{m}$ to $r$ is bounded by $\frac{4 \lambda}{\lambda-1}$ as in the preceding situation.

Now suppose that $\frac{1}{2} \theta \alpha \leq \varepsilon$ and $\frac{1}{m}<\varepsilon$. Let $B$ be a number such that $\alpha+\varepsilon=$ $\zeta(B+\alpha+\varepsilon)$. We see that $B=\frac{1-\zeta}{\zeta}(\alpha+\varepsilon)$ is that number. Since we can assume that $\theta<\zeta$, then $B<A$. Indeed we will assume $\theta<\frac{1}{2}$ and $\zeta>\frac{1}{2}$. So, we have that $x+\frac{1}{m}<\xi+A$, because $A=\frac{1-\theta}{\theta}(\alpha+\varepsilon)>\alpha+\varepsilon \geq \varepsilon>\frac{1}{m}$. But, it may happen that $\xi+B \leq x+\frac{1}{m}$, because $\alpha$ can be even zero. Let's see first this special case, 
i.e. if $\frac{1}{2} \theta \alpha \leq \varepsilon$ and $\alpha+B \leq \frac{1}{m}<\varepsilon$. We do the following:

$$
\begin{aligned}
\left|\int_{0}^{x-\varepsilon} e^{i f(t)} \frac{1}{t-x} d t\right| & \leq \frac{4}{\varepsilon f^{\prime}(x-\varepsilon)}=\frac{4 \lambda}{\lambda-1} \\
\left|\int_{x-\varepsilon}^{x-\frac{1}{m}} e^{i f(t)} \frac{1}{t-x} d t\right| & \leq m \varepsilon \leq \frac{\varepsilon}{\alpha+B} \leq \frac{\zeta}{1-\zeta} \\
\left|\int_{x+\frac{1}{m}}^{\xi+A} e^{i f(t)} \frac{1}{t-x} d t\right| & \leq \log \left(\frac{\alpha+A}{\frac{1}{m}}\right) \leq \log \left(\frac{\alpha+A}{\alpha+B}\right) \\
& \leq \log \left[\left(\frac{1-\theta}{\theta}\right)\left(\frac{\zeta}{1-\zeta}\right)\right] .
\end{aligned}
$$

(As before, we can assume $\xi+A<r$. Indeed, since $\frac{1}{2} \theta \alpha \leq \varepsilon$, then $\xi+A<r$ if $x$ is sufficiently small.)

$$
\left|\int_{\xi+A}^{r} e^{i f(t)} \frac{1}{t-x} d t\right| \leq \frac{4}{(\alpha+A)\left|f^{\prime}(\xi+A)\right|} \leq \frac{4}{\varepsilon f^{\prime}(x-\varepsilon)}=\frac{4 \lambda}{\lambda-1}
$$

by (17).

Let's see now the case that was left over, i.e. we assume $\frac{1}{2} \theta \alpha \leq \varepsilon, \frac{1}{m}<\varepsilon$ and $\frac{1}{m}<\alpha+B$. We claim that

$$
n \varphi^{\prime}(\xi+B)-m \leq M\left[m-n \varphi^{\prime}(x-\varepsilon)\right] .
$$

To prove (18) we use condition (5). By the choice of $B$ we have that $\xi=$ $(1-\zeta)(x-\varepsilon)+\zeta(\xi+B)$. Also, since $\varepsilon f^{\prime}(x-\varepsilon)=\frac{\lambda-1}{\lambda}$ and $\varepsilon m>1$, then $\varphi^{\prime}(\xi)<\lambda \varphi^{\prime}(x-\varepsilon)$. Hence, (18) follows from (5). Now, let's see how to get boundedness of the integral in that last situation. From zero to $x-\varepsilon$ is quite easy and we have $\left|\int_{o}^{x-\varepsilon} e^{i f(t)} \frac{1}{t-x} d t\right| \leq \frac{4}{\varepsilon f^{\prime}(x-\varepsilon)}=\frac{4 \lambda}{\lambda-1}$. (We recall again here that we are free to choose the number $\tau$. So, let's choose $\tau$ sufficiently small in order to have $\xi+B<r$. This procedure is not quite necessary, but it simplifies a little bit that part of the proof. Note that it is possible to choose such $\tau$, for in that situation we have

$$
\begin{aligned}
\xi+B=x+\alpha+\frac{1-\zeta}{\zeta}(\alpha+\varepsilon) & \leq x+\frac{2}{\theta} \varepsilon+\frac{1-\zeta}{\zeta}\left(\frac{2}{\theta} \varepsilon+\varepsilon\right) \\
& \leq x+\frac{2}{\theta} x+\frac{1-\zeta}{\zeta}\left(\frac{2}{\theta} x+x\right) \\
& \leq\left(\frac{4}{\theta}+2\right) x .
\end{aligned}
$$

So, $\tau<\frac{1}{6} \theta r$ will do the job.)

$$
\left|\int_{\xi+B}^{\xi+A} e^{i f(t)} \frac{1}{t-x} d t\right| \leq \log \left(\frac{\alpha+A}{\alpha+B}\right) \leq \log \left[\left(\frac{1-\theta}{\theta}\right)\left(\frac{\zeta}{1-\zeta}\right)\right] .
$$

We can also assume that $\xi+A<r$, because if not, we get boundedness of the integral over the interval $[\xi+B, r]$ as we did above. So,

$$
\left|\int_{\xi+A}^{r} e^{i f(t)} \frac{1}{t-x} d t\right|=\frac{4}{(\alpha+A)\left|f^{\prime}(\xi+A)\right|} \leq \frac{4}{\varepsilon f^{\prime}(x-\varepsilon)}=\frac{4 \lambda}{\lambda-1},
$$

by (17). 
It remains to prove boundedness over $\left[x-\varepsilon, x-\frac{1}{m}\right] \cup\left[x+\frac{1}{m}, \xi+B\right]$. We will use cancellation around $x$ to do this. The cancellation depends on the inequality (18) as well as on the assumption that $\varepsilon \geq \frac{1}{2} \theta \alpha$. We have

$$
\begin{gathered}
\quad\left|\int_{x-\varepsilon}^{x-\frac{1}{m}} e^{i f(t)} \frac{1}{t-x} d t+\int_{x+\frac{1}{m}}^{\xi+B} e^{i f(t)} \frac{1}{t-x} d t\right| \\
=\mid \int_{x-\varepsilon}^{x-\frac{1}{m}}\left(e^{i f(t)}-e^{i f(x)}\right) \frac{1}{t-x} d t+\int_{x+\frac{1}{m}}^{\xi+B}\left(e^{i f(t)}-e^{i f(x)}\right) \frac{1}{t-x} d t \\
+\int_{x-\varepsilon}^{x-\frac{1}{m}} e^{i f(x)} \frac{1}{t-x} d t+\int_{x+\frac{1}{m}}^{\xi+B} e^{i f(x)} \frac{1}{t-x} d t \mid \\
\leq \varepsilon f^{\prime}(x-\varepsilon)+M f^{\prime}(x-\varepsilon)(\alpha+B)+\left|\int_{1 / m}^{\alpha+B} \frac{1}{t} d t-\int_{1 / m}^{\varepsilon} \frac{1}{t} d t\right| \\
\leq \varepsilon f^{\prime}(x-\varepsilon)+M \varepsilon f^{\prime}(x-\varepsilon)\left[\frac{2}{\theta}+\frac{1-\zeta}{\zeta}\left(\frac{2}{\theta}+1\right)\right]+\left|\log \left(\frac{\alpha+B}{\varepsilon}\right)\right| \\
=\frac{\lambda-1}{\lambda}+M\left(\frac{\lambda-1}{\lambda}\right)\left[\frac{2}{\theta}+\frac{1-\zeta}{\zeta}\left(\frac{2}{\theta}+1\right)\right]+\left|\log \left(\frac{\alpha+B}{\varepsilon}\right)\right|,
\end{gathered}
$$

by (18).

Since $\frac{\alpha+B}{\varepsilon} \geq \frac{B}{\varepsilon}=\frac{\left(\frac{1-\zeta}{\zeta}\right)(\alpha+\varepsilon)}{\varepsilon} \geq \frac{1-\zeta}{\zeta}$ and $\frac{\alpha+B}{\varepsilon}<\frac{\frac{2}{\theta} \varepsilon+\left(\frac{1-\zeta}{\zeta}\right)\left(\frac{2}{\theta} \varepsilon+\varepsilon\right)}{\varepsilon} \leq \frac{4+\theta}{\theta}$, then $\left|\log \left(\frac{\alpha+B}{\varepsilon}\right)\right| \leq \max \left\{\left|\log \left(\frac{1-\zeta}{\zeta}\right)\right|, \log \left(\frac{4+\theta}{\theta}\right)\right\}$. This concludes the case $x \leq \xi$.

Assume now that $\xi<x$. Call $\beta=x-\xi$. This case is quite different from the previous one, and it requires different arguments. We will introduce a new number, say $\delta$, and that number controls the amount of oscillation and the amount of cancellation that we have. The control is done in the following sense: when $\delta$ becomes small, this means that we lose cancellation but we gain oscillation; and when $\delta$ becomes large this means that we lose oscillation, but we gain cancellation.

Let $\delta$ be a positive number such that $\delta\left[n \varphi^{\prime}\left(\xi+\frac{1+\zeta}{2} \beta\right)-m\right]=\lambda-1$. Since $f^{\prime}(x-\varepsilon)>0$, then $x-\varepsilon<\xi$. So, $\varepsilon>\beta$. Let's consider first the following situation. Suppose that $0<\frac{1}{m}<\delta<\frac{1-\zeta}{2} \beta$ and $\varepsilon \leq \frac{1+\zeta}{1-\zeta} \beta$. We claim that

$$
n \varphi^{\prime}\left(\xi+\frac{1+\zeta}{2 \zeta} \beta\right)-m \leq(M+1)\left[n \varphi^{\prime}\left(\xi+\frac{1+\zeta}{2} \beta\right)-m\right] .
$$

To prove (19) we use condition (5). (Note that we can choose $\tau$ sufficiently small in order to have $\xi+\frac{1+\zeta}{2 \zeta} \beta<r$.) We have that $\xi+\frac{1+\zeta}{2} \beta=(1-\zeta) \xi+\zeta\left(\xi+\frac{1+\zeta}{2 \zeta} \beta\right)$. Since $\delta\left|f^{\prime}\left(\xi+\frac{1+\zeta}{2} \beta\right)\right|=\lambda-1$ and $\delta m>1$, then $\varphi^{\prime}\left(\xi+\frac{1+\zeta}{2} \beta\right)<\lambda \varphi^{\prime}(\xi)$. Hence, (19) follows from (5). Let's work with the integral now. We divide it in several parts.

$$
\left|\int_{0}^{x-\varepsilon} e^{i f(t)} \frac{1}{t-x} d t\right| \leq \frac{4}{\varepsilon f^{\prime}(x-\varepsilon)}=\frac{4 \lambda}{\lambda-1},
$$




$$
\begin{gathered}
\left|\int_{x-\varepsilon}^{\xi+\frac{1+\zeta}{2} \beta} e^{i f(t)} \frac{1}{t-x} d t\right| \leq \log \left(\frac{\varepsilon}{\frac{1-\zeta}{2} \beta}\right) \leq \log \left(\frac{2(1+\zeta)}{(1-\zeta)^{2}}\right), \\
\left|\int_{\xi+\frac{1+\zeta}{2} \beta}^{x-\delta} e^{i f(t)} \frac{1}{t-x} d t\right| \leq \frac{4}{\delta\left|f^{\prime}\left(\xi+\frac{1+\zeta}{2} \beta\right)\right|}=\frac{4}{\lambda-1},
\end{gathered}
$$

and

$$
\left|\int_{x+\delta}^{r} e^{i f(t)} \frac{1}{t-x} d t\right| \leq \frac{4}{\delta\left|f^{\prime}(x+\delta)\right|} \leq \frac{4}{\delta\left|f^{\prime}\left(\xi+\frac{1+\zeta}{2} \beta\right)\right|}=\frac{4}{\lambda-1} .
$$

To prove boundedness around $x$ we use the small amount of cancellation that we have there:

$$
\begin{gathered}
\left|\int_{x-\delta}^{x-\frac{1}{m}} e^{i f(t)} \frac{1}{t-x} d t+\int_{x-\frac{1}{m}}^{x+\delta} e^{i f(t)} \frac{1}{t-x} d t\right| \\
=\left|\int_{x-\delta}^{x-\frac{1}{m}}\left(e^{i f(t)}-e^{i f(x)}\right) \frac{1}{t-x} d t+\int_{x+\frac{1}{m}}^{x+\delta}\left(e^{i f(t)}-e^{i f(x)}\right) \frac{1}{t-x} d t\right| \\
\leq 2 \delta\left[n \varphi^{\prime}\left(\xi+\frac{1+\zeta}{2 \zeta} \beta\right)-m\right] \\
\leq 2 \delta(M+1)\left[n \varphi^{\prime}\left(\xi+\frac{1+\zeta}{2} \beta\right)-m\right]=2(M+1)(\lambda-1),
\end{gathered}
$$

by (19).

Another possible situation is that: $0<\delta \leq \frac{1}{m}<\frac{1-\zeta}{2} \beta$ and $\varepsilon \leq \frac{1+\zeta}{1-\zeta} \beta$. This case is better than the previous one because the small $\delta$ here means that we have a large amount of oscillation, and $\frac{1}{m} \geq \delta$ means that $\frac{1}{m}$ is large enough to put us away from the bad singularity at $x$. Hence, as we shall see, we don't need conditions (4) and (5) in this case. We have:

$$
\begin{aligned}
\left|\int_{0}^{x-\varepsilon} e^{i f(t)} \frac{1}{t-x} d t\right| & \leq \frac{4}{\varepsilon f^{\prime}(x-\varepsilon)}=\frac{4 \lambda}{\lambda-1} \\
\left|\int_{x-\varepsilon}^{\xi+\frac{1+\zeta}{2} \beta} e^{i f(t)} \frac{1}{t-x} d t\right| & \leq \log \left(\frac{\varepsilon}{\frac{1-\zeta}{2} \beta}\right) \leq \log \left(\frac{2(1+\zeta)}{(1-\zeta)^{2}}\right) \\
\left|\int_{\xi+\frac{1+\zeta}{2} \beta}^{x-\frac{1}{m}} e^{i f(t)} \frac{1}{t-x} d t\right| & \leq \frac{4 m}{\left|f^{\prime}\left(\xi+\frac{1+\zeta}{2} \beta\right)\right|} \\
& \leq \frac{4}{\delta\left|f^{\prime}\left(\xi+\frac{1+\zeta}{2} \beta\right)\right|}=\frac{4}{\lambda-1} \\
\left|\int_{x+\frac{1}{m}}^{r} e^{i f(t)} \frac{1}{t-x} d t\right| & \leq \frac{4 m}{\left|f^{\prime}\left(x+\frac{1}{m}\right)\right|} \\
& \leq \frac{4}{\delta\left|f^{\prime}\left(\xi+\frac{1+\zeta}{2} \beta\right)\right|}=\frac{4}{\lambda-1}
\end{aligned}
$$


The next situation is: $0<\delta<\frac{1-\zeta}{2} \beta \leq \frac{1}{m}$ and $\varepsilon \leq \frac{1+\zeta}{1-\zeta} \beta$. In that case we do the following: if $x-\frac{1}{m} \leq x-\varepsilon$, then

$$
\begin{aligned}
\left|\int_{0}^{x-\frac{1}{m}} e^{i f(t)} \frac{1}{t-x} d t\right| & \leq \frac{4 m}{f^{\prime}\left(x-\frac{1}{m}\right)} \leq \frac{4}{\varepsilon f^{\prime}(x-\varepsilon)}=\frac{4 \lambda}{\lambda-1}, \\
\left|\int_{x+\frac{1}{m}}^{r} e^{i f(t)} \frac{1}{t-x} d t\right| & \leq \frac{4 m}{\left|f^{\prime}\left(x+\frac{1}{m}\right)\right|} \leq \frac{4}{\delta\left|f^{\prime}\left(\xi+\frac{1+\zeta}{2} \beta\right)\right|}=\frac{4}{\lambda-1} .
\end{aligned}
$$

If $x-\varepsilon<x-\frac{1}{m}$, then

$$
\begin{aligned}
\left|\int_{0}^{x-\varepsilon} e^{i f(t)} \frac{1}{t-x} d t\right| & \leq \frac{4}{\varepsilon f^{\prime}(x-\varepsilon)}=\frac{4 \lambda}{\lambda-1} \\
\left|\int_{x-\varepsilon}^{x-\frac{1}{m}} e^{i f(t)} \frac{1}{t-x} d t\right| & \leq \log (\varepsilon m) \leq \log \left(\frac{2(1+\zeta)}{(1-\zeta)^{2}}\right) \\
\left|\int_{x+\frac{1}{m}}^{r} e^{i f(t)} \frac{1}{t-x} d t\right| & \leq \frac{4 m}{\left|f^{\prime}\left(x+\frac{1}{m}\right)\right|} \\
& \leq \frac{4}{\delta\left|f^{\prime}\left(\xi+\frac{1+\zeta}{2} \beta\right)\right|}=\frac{4}{\lambda-1} .
\end{aligned}
$$

Suppose now that $\frac{1}{m}<\frac{1-\zeta}{2} \beta \leq \delta$ and $\varepsilon \leq \frac{1+\zeta}{1-\zeta} \beta$. In that situation we have to use conditions (4) and (5) because the large $\delta$ means that we don't have enough oscillation, and the small $\frac{1}{m}$ means that we are too close to the singularity at $x$. But, on the other hand, the large $\delta$ means that we have much cancellation around $x$, and we will use that fact to compensate the lack of oscillation in this case.

$$
\begin{gathered}
\left|\int_{0}^{x-\varepsilon} e^{i f(t)} \frac{1}{t-x} d t\right| \leq \frac{4}{\varepsilon f^{\prime}(x-\varepsilon)}=\frac{4 \lambda}{\lambda-1}, \\
\left|\int_{x-\varepsilon}^{\xi+\frac{1+\zeta}{2} \beta} e^{i f(t)} \frac{1}{t-x} d t\right| \leq \log \left(\frac{\varepsilon}{\frac{1-\zeta}{2} \beta}\right) \\
\leq \log \left(\frac{2(1+\zeta)}{(1-\zeta)^{2}}\right), \\
\left|\int_{\xi+\frac{1+\zeta}{2} \beta}^{x-\frac{1}{m}} e^{i f(t)} \frac{1}{t-x} d t+\int_{x+\frac{1}{m}}^{x+\frac{1-\zeta}{2} \beta} e^{i f(t)} \frac{1}{t-x} d t\right| \\
=\left|\int_{\xi+\frac{1+\zeta}{2} \beta}^{x-\frac{1}{m}}\left(e^{i f(t)}-e^{i f(x)}\right) \frac{1}{t-x} d t+\int_{x+\frac{1}{m}}^{x+\frac{1-\zeta}{2} \beta}\left(e^{i f(t)}-e^{i f(x)}\right) \frac{1}{t-x} d t\right| \\
\leq 2 \delta\left|f^{\prime}\left(\xi+\frac{1+\zeta}{2 \zeta} \beta\right)\right| \\
\leq 2 \delta(M+1)\left|f^{\prime}\left(\xi+\frac{1+\zeta}{2} \beta\right)\right|=2(M+1)(\lambda-1),
\end{gathered}
$$

by (19).

We still have to prove boundedness of the integral over the interval $\left[x+\frac{1-\zeta}{2} \beta, r\right]$. Since $\delta$ is large, then we may not have enough oscillation at the point $x+\frac{1-\zeta}{2} \beta$. 
So, we cannot just integrate from $x+\frac{1-\zeta}{2} \beta$ to $r$ using the Van der Corput lemma. We have to use condition (4).

Let $A^{\prime}$ be a number such that $\varepsilon-\beta=\theta\left(A^{\prime}+\varepsilon-\beta\right)$. We can see that $A^{\prime}=$ $\frac{1-\theta}{\theta}(\varepsilon-\beta)$ is that number. We claim that

$$
\begin{array}{r}
n \varphi^{\prime}\left(x+\frac{1-\zeta}{2} \beta+A^{\prime}\right)-m \geq n \varphi^{\prime}\left(\xi+A^{\prime}\right)-m, \\
n \varphi^{\prime}\left(\xi+A^{\prime}\right)-m \geq m-n \varphi^{\prime}(x-\varepsilon) .
\end{array}
$$

To prove (20) we use (4). By the choice of $A^{\prime}$ we have that $\xi=(1-\theta)(x-\varepsilon)$ $+\theta\left(\xi+A^{\prime}\right)$. Since $\xi+A^{\prime}<x+\frac{1-\zeta}{2} \beta+A^{\prime},(20)$ follows from (4).

We will use inequality (20) to show boundedness over the interval $\left[x+\frac{1-\zeta}{2} \beta, r\right]$. We divide the interval from $x+\frac{1-\zeta}{2} \beta$ to $x+\frac{1-\zeta}{2} \beta+A^{\prime}$ and from $x+\frac{1-\zeta}{2} \beta+A^{\prime}$ to $r$. ( Note that we can choose $\tau$ sufficiently small in order to have $x+\frac{1-\zeta}{2} \beta+A^{\prime}<r$, but again this is not quite necessary because if not we just integrate from $x+\frac{1-\zeta}{2} \beta$ to $r$ as we have done before. ) We have

$$
\left|\int_{x+\frac{1-\zeta}{2} \beta}^{x+\frac{1-\zeta}{2} \beta+A^{\prime}} e^{i f(t)} \frac{1}{t-x} d t\right| \leq \log \left(\frac{\frac{1-\zeta}{2} \beta+A^{\prime}}{\frac{1-\zeta}{2} \beta}\right) \leq \log \left(\frac{\frac{1-\zeta}{2}+\frac{1-\theta}{\theta}\left(\frac{1+\zeta}{1-\zeta}\right)}{\frac{1-\zeta}{2}}\right)
$$

and

$$
\begin{aligned}
\left|\int_{x+\frac{1-\zeta}{2} \beta+A^{\prime}}^{r} e^{i f(t)} \frac{1}{t-x} d t\right| & \leq \frac{4}{\left|f^{\prime}\left(x+\frac{1-\zeta}{2} \beta+A^{\prime}\right)\right|\left(\frac{1-\zeta}{2} \beta+A^{\prime}\right)} \\
& \leq \frac{4}{\left(\frac{1-\zeta}{2} \beta\right) f^{\prime}(x-\varepsilon)}
\end{aligned}
$$

by $(20)$.

But now, since $\varepsilon \leq \frac{1+\zeta}{1-\zeta} \beta$, then $\frac{1-\zeta}{2} \beta \geq\left(\frac{1-\zeta}{2}\right)\left(\frac{1-\zeta}{1+\zeta}\right) \varepsilon$. Hence,

$$
\begin{aligned}
\left(\frac{1-\zeta}{2} \beta\right) f^{\prime}(x-\varepsilon) & \geq\left(\frac{1-\zeta}{2}\right)\left(\frac{1-\zeta}{1+\zeta}\right) \varepsilon f^{\prime}(x-\varepsilon) \\
& =\left(\frac{\lambda-1}{\lambda}\right)\left(\frac{1-\zeta}{2}\right)\left(\frac{1-\zeta}{1+\zeta}\right)
\end{aligned}
$$

and we are done. (Note that in order to bound the above integral over $\left[x+\frac{1-\zeta}{2} \beta, r\right]$ we could have worked with the point $x+\varepsilon+A^{\prime}$ instead of $x+\frac{1-\zeta}{2} \beta+A^{\prime}$.)

The next situation is the following. Assume that $\frac{1-\zeta}{2} \beta \leq \frac{1}{m}, \frac{1-\zeta}{2} \beta \leq \delta$ and $\varepsilon \leq \frac{1+\zeta}{1-\zeta} \beta$. This case is a little better than the previous one because the large $\frac{1}{m}$ means that we are far away from the singularity at $x$. But since $\delta$ can be still large, we might not have enough oscillation. So we still have to use condition (4). If $x-\frac{1}{m} \leq x-\varepsilon$, then

$$
\left|\int_{0}^{x-\frac{1}{m}} e^{i f(t)} \frac{1}{t-x} d t\right| \leq \frac{4 m}{f^{\prime}(x-\varepsilon)} \leq \frac{4}{\varepsilon f^{\prime}(x-\varepsilon)}=\frac{4 \lambda}{\lambda-1} .
$$


If $x-\frac{1}{m}>x-\varepsilon$, then

$$
\left|\int_{0}^{x-\varepsilon} e^{i f(t)} \frac{1}{t-x} d t\right| \leq \frac{4}{\varepsilon f^{\prime}(x-\varepsilon)}=\frac{4 \lambda}{\lambda-1}
$$

and

$$
\left|\int_{x-\varepsilon}^{x-\frac{1}{m}} e^{i f(t)} \frac{1}{t-x} d t\right| \leq \log (\varepsilon m) \leq \log \left(\frac{2(1+\zeta)}{(1-\zeta)^{2}}\right) .
$$

This proves boundedness over the interval $\left[0, x-\frac{1}{m}\right]$. To work out the integral over $\left[x+\frac{1}{m}, r\right]$ we do the following:

$$
\begin{aligned}
\left|\int_{x+\frac{1}{m}}^{x+\frac{1}{m}+A^{\prime}} e^{i f(t)} \frac{1}{t-x} d t\right| & \leq \log \left(\frac{\frac{1}{m}+A^{\prime}}{\frac{1}{m}}\right) \leq\left(1+\frac{\left(\frac{1-\theta}{\theta}\right)\left(\frac{1+\zeta}{1-\zeta}\right)}{\left(\frac{1-\zeta}{2}\right)}\right) \\
\text { and } & \\
\left|\int_{x+\frac{1}{m}+A^{\prime}}^{r} e^{i f(t)} \frac{1}{t-x} d t\right| & \leq \frac{4 m}{\left|f^{\prime}\left(x+\frac{1}{m}+A^{\prime}\right)\right|} \\
& \leq \frac{4}{\frac{1-\zeta}{2} \beta\left|f^{\prime}\left(x+\frac{1-\zeta}{2} \beta+A^{\prime}\right)\right|} \\
& \leq \frac{4}{\left(\frac{1-\zeta}{2}\right)\left(\frac{1-\zeta}{1+\zeta}\right) \varepsilon f^{\prime}(x-\varepsilon)}=\frac{4}{\left(1-\frac{1}{\lambda}\right)\left(\frac{1-\zeta}{2}\right)\left(\frac{1-\zeta}{1+\zeta}\right)},
\end{aligned}
$$

by $(20)$.

So far we studied all possible situations with $\varepsilon \leq \frac{1+\zeta}{1-\zeta} \beta$. We still have to analyse the problem when $\varepsilon>\frac{1+\zeta}{1-\zeta} \beta$. For this, let $B^{\prime}$ be a number such that $\varepsilon-\beta=$ $\zeta\left(B^{\prime}+\varepsilon-\beta\right)$. We see that $B^{\prime}=\frac{1-\zeta}{\zeta}(\varepsilon-\beta)$ is that number.

Suppose we have the following situation : $\frac{1}{m}<B^{\prime}-\beta$ and $\varepsilon>\frac{1+\zeta}{1-\zeta} \beta$. (Note that $B^{\prime}>2 \beta$ since $\varepsilon>\frac{1+\zeta}{1-\zeta} \beta$.) Since $\frac{1}{m}<B^{\prime}-\beta$, then $x+\frac{1}{m}<\xi+B^{\prime}$. Also, $B^{\prime}-\beta \leq B^{\prime}+\frac{1-\zeta}{\zeta}(\varepsilon-\beta) \leq \varepsilon-\beta<\varepsilon$. So, $\frac{1}{m}<B^{\prime}-\beta$ implies that $\frac{1}{m}<\varepsilon$. We claim that

$$
n \varphi^{\prime}\left(\xi+B^{\prime}\right)-m \leq M\left[m-n \varphi^{\prime}(x-\varepsilon)\right] .
$$

To prove (21) we use condition (5). By the choice of $B^{\prime}$ we have that $\xi=$ $(1-\zeta)(x-\varepsilon)+\zeta\left(\xi+B^{\prime}\right)$. Since $\varepsilon f^{\prime}(x-\varepsilon)=\frac{\lambda-1}{\lambda}$ and $\varepsilon m>1, \varphi^{\prime}(\xi) \leq \lambda \varphi^{\prime}(x-\varepsilon)$. Hence, (21) follows from (5).

Now, let's estimate the integral. We have

$$
\begin{gathered}
\left|\int_{0}^{x-\varepsilon} e^{i f(t)} \frac{1}{t-x} d t\right| \leq \frac{4}{\varepsilon f^{\prime}(x-\varepsilon)}=\frac{4 \lambda}{\lambda-1}, \\
\left|\int_{x-\varepsilon}^{x-\frac{1}{m}} e^{i f(t)} \frac{1}{t-x} d t+\int_{x+\frac{1}{m}}^{\xi+B^{\prime}} e^{i f(t)} \frac{1}{t-x} d t\right| \\
\leq M \varepsilon f^{\prime}(x-\varepsilon)+M\left(B^{\prime}-\beta\right) f^{\prime}(x-\varepsilon)+\left|\int_{1 / m}^{B^{\prime}-\beta} \frac{1}{t} d t-\int_{1 / m}^{\varepsilon} \frac{1}{t} d t\right|
\end{gathered}
$$




$$
\leq 2 M \varepsilon f^{\prime}(x-\varepsilon)+\log \left(\frac{\varepsilon}{B^{\prime}-\beta}\right) \leq 2 M\left(\frac{\lambda-1}{\lambda}\right)+\log \left(\frac{1+\zeta}{1-\zeta}\right),
$$

by (21).

$$
\begin{aligned}
\left|\int_{\xi+B^{\prime}}^{\xi+A^{\prime}} e^{i f(t)} \frac{1}{t-x} d t\right| & \leq \log \left(\frac{A^{\prime}-\beta}{B^{\prime}-\beta}\right) \\
& \leq \log \left(\frac{2 A^{\prime}}{B^{\prime}}\right)=\log \left[\left(\frac{1-\theta}{\theta}\right)\left(\frac{2 \zeta}{1-\zeta}\right)\right] . \\
\left|\int_{\xi+A^{\prime}}^{r} e^{i f(t)} \frac{1}{t-x} d t\right| & \leq \frac{4}{\left(A^{\prime}-\beta\right)\left|f^{\prime}\left(\xi+A^{\prime}\right)\right|} \leq \frac{4}{\left(B^{\prime}-\beta\right) f^{\prime}(x-\varepsilon)} \\
& \leq \frac{8}{B^{\prime} f^{\prime}(x-\varepsilon)} \leq \frac{4(1+\zeta) \lambda}{(\lambda-1)(1-\zeta)}
\end{aligned}
$$

by (20).

Finally, the last situation is that: $B^{\prime}-\beta \leq \frac{1}{m}$ and $\varepsilon>\frac{1+\zeta}{1-\zeta} \beta$. If $x-\frac{1}{m} \leq x-\varepsilon$, then

$$
\left|\int_{0}^{x-\frac{1}{m}} e^{i f(t)} \frac{1}{t-x} d t\right| \leq \frac{4 m}{f^{\prime}\left(x-\frac{1}{m}\right)} \leq \frac{4}{\varepsilon f^{\prime}(x-\varepsilon)}=\frac{4 \lambda}{\lambda-1}
$$

If $x-\frac{1}{m}>x-\varepsilon$, then

$$
\begin{aligned}
&\left|\int_{0}^{x-\varepsilon} e^{i f(t)} \frac{1}{t-x} d t\right| \leq \frac{4}{\varepsilon f^{\prime}(x-\varepsilon)}=\frac{4 \lambda}{\lambda-1} \\
& \text { and } \\
&\left|\int_{x-\varepsilon}^{x-\frac{1}{m}} e^{i f(t)} \frac{1}{t-x} d t\right| \leq \log (\varepsilon m) \leq \log \left(\frac{\varepsilon}{B^{\prime}-\beta}\right) \\
& \leq \log \left(\frac{1+\zeta}{1-\zeta}\right) .
\end{aligned}
$$

This proves boundedness of the integral over $\left[0, x-\frac{1}{m}\right]$. To estimate the integral on $\left[x+\frac{1}{m}, r\right]$ we do as follows: if $x+\frac{1}{m} \geq \xi+A^{\prime}$, then

$$
\begin{aligned}
\left|\int_{x+\frac{1}{m}}^{r} e^{i f(t)} \frac{1}{t-x} d t\right| & \leq \frac{4 m}{\left|f^{\prime}\left(x+\frac{1}{m}\right)\right|} \leq \frac{4}{\left(B^{\prime}-\beta\right)\left|f^{\prime}\left(\xi+A^{\prime}\right)\right|} \\
& \leq \frac{4}{\left(\frac{1-\zeta}{1+\zeta}\right) \varepsilon f^{\prime}(x-\varepsilon)}=\frac{4 \lambda(1+\zeta)}{(\lambda-1)(1-\zeta)}
\end{aligned}
$$

by (20). 
If $x+\frac{1}{m}<\xi+A^{\prime}$, then

$$
\begin{aligned}
\left|\int_{x+\frac{1}{m}}^{\xi+A^{\prime}} e^{i f(t)} \frac{1}{t-x} d t\right| & \leq \log \left(\frac{A^{\prime}-\beta}{\frac{1}{m}}\right) \leq \log \left(\frac{A^{\prime}}{B^{\prime}-\beta}\right) \\
& \leq \log \left(\frac{2 A^{\prime}}{B^{\prime}}\right)=\log \left(\frac{2 \zeta(1-\theta)}{\theta(1-\zeta)}\right)
\end{aligned}
$$

and

$$
\begin{aligned}
\left|\int_{\xi+A^{\prime}}^{r} e^{i f(t)} \frac{1}{t-x} d t\right| & \leq \frac{4}{\left(A^{\prime}-\beta\right)\left|f^{\prime}\left(\xi+A^{\prime}\right)\right|} \leq \frac{8}{B^{\prime} f^{\prime}(x-\varepsilon)} \\
& \leq \frac{4}{\left(\frac{1-\zeta}{1+\zeta}\right) \varepsilon f^{\prime}(x-\varepsilon)}=\frac{4 \lambda(1+\zeta)}{(\lambda-1)(1-\zeta)}
\end{aligned}
$$

by $(20)$.

This concludes the proof of the theorem.

\section{REFERENCES}

1. Kahane, J. P. - Quatre Leçons sur les Homéomorphismes du Circle et les Series de Fourier. Topics in Modern Harmonic Analysis, Proceedings of a seminar held in Torino and Milano, May - June 1982, vol. II, Roma, 1983. MR 85k:42012

2. Stein, E. M. and Wainger, S. - The Estimation of an Integral Arising in Multiplier Transformations, Studia Math. 35 (1970), 101 - 104. MR 42:904

3. Stein, E. M. - Oscillatory Integral in Fourier Analysis, Beijing Lectures in Harmonic Analysis, edited by E. M. Stein, Princeton University Press. (1986), 307 - 355. MR 88g:42022

4. Nagel, A., Vance, J., Wainger, S. and Weinberg, D. - Hilbert Transforms for Convex Curves, Duke Mathematical Journal. Vol. 50 (1983), 735 - 744. MR 85a:42025

5. Santos Zumpano, A. - Homeomorphisms Of The Circle And Fourier Series, Ph.D thesis University of Wisconsin - Madison, May 1987.

Departamento de Matematica, Universidade Federal de Minas Gerais, Caixa Postal 702, 30.123-970 Belo Horizonte MG, Brazil

E-mail address: zumpano@mat.ufmg.br 